\section{EARLY SEQUENTIAL COMBINATION THERAPY WITH MIZORIBINE AND TACROLIMUS IN SIXTY THREE PATIENTS OF LUPUS NEPHRITIS IN A SINGLE CENTRECENTER IN JAPAN}

M Okada*, R Kawato, R Rokutanda. St. Luke's International Hospital, ImmunoRheumatology Centre, Chuo-ku, Japan

10.1136/lupus-2017-000215.232

Background and aims Mizoribine is an inhibitor of inosine monophosphate dehydrogenase, which is widely used for patients with lupus nephritis and also patients after renal transplants. Its anti-cytomegaloviral effect is unique as an immunosuppressant. We examined the efficacy and safety of early sequential combination of mizoribine and tacrolimus in lupus nephritis.

Methods Retrospective review of electric medical record was performed for all the 63 patients who received the combination therapy of mizoribine and tacrolimus and corticosteroids for induction or maintenance of lupus nephritis at St. Luke's International Hospital, Tokyo, Japan. For efficacy analysis, we extracted a series of change in serum creatinine, serum complement level, urine protein creatinine ratio, dose of corticosteroid. We further reviewed safety profile such as adverse events occurred during the use of multi-target therapy, drug survival rate, or reasons for discontinue multi-target therapy in all patients. Complete remission of lupus nephritis was defined as a value of proteinuria $<0.5 \mathrm{~g} / \mathrm{gCr}$, normal urinary sediment, serum albumin $3.5 \mathrm{~g} / \mathrm{dl}$ and a normal value of serum creatinine.

Results Fifty six out of the sixty three patients (female: male $=59: 4$, average age 37.4 years old) achieved complete remission in 6 months and there were only two relapses and both of them had Class $\mathrm{V}$ nephritis. At four month, the average urine protein creatinine ratio was $0.36 \mathrm{~g} / \mathrm{gCr}$, and the average dose of prednisolone was $9.9 \mathrm{mg} /$ day. There were only three episodes of infections which required antibiotics administrations.

Conclusions Early sequential combination of mizoribine and tacrolimus seems to be effective and safe for lupus nephritis.

\section{DEFINING SUBSETS IN SLE: THE INTERPLAY AMONG IFN- $\lambda 1$, IFN- $\alpha$ AND TH17 AXIS CYTOKINES}

${ }^{1} \mathrm{~V}$ Oke*, ${ }^{2} \mathrm{~S}$ Brauner, ${ }^{3} \mathrm{~A}$ Larsson, ${ }^{1} \mathrm{~J}$ Gustafsson, ${ }^{1} \mathrm{~A}$ Zickert, ${ }^{1} \mathrm{I}$ Gunnarsson, ${ }^{1} \mathrm{E}$ Svenungsson. ${ }^{1}$ Karolinska Institutet, Department of Medicine- Rheumatology Clinic, Stockholm, Sweden; ${ }^{2}$ Karolinska Institutet, Department of Medicine- Rheumatology research lab, Stockholm, Sweden; ${ }^{3}$ Uppsala University, Department of Medical Sciences- Clinical Chemistry, Uppsala, Sweden

\subsection{6/lupus-2017-000215.233}

Background and aims Interferon (IFN)- $\alpha$ s are pivotal in systemic lupus erythematosus (SLE), and type III IFNs (IFN- $\lambda s$ ) were recently also associated with SLE. We investigated levels of IFN- $\alpha$ and IFN- $\lambda 1$, and related cytokines in SLE patients and controls.

Methods We included 261 SLE patients and 261 population controls. All were examined and assessed for current organ manifestations and disease activity/damage using SLAM, SLEDAI and ACR/SDI scales. Levels of IFN- $\lambda 1$, IFN- $\alpha$, IL-17A, IL-23 and IP-10 were measured by ELISA.

Results IFN- $\lambda 1$ and IFN- $\alpha$ were detected in $29 \%$ and $44 \%$ of patients respectively, but their levels did not correlate. High serum levels of IFN- $\lambda 1$ were positively associated with antinucleosome antibodies and lymphopenia, but negatively with musculoskeletal damage. Positive correlations between levels of IFN- $\lambda 1$, IL-17A and IL-23 were observed. Patients with high levels of these three cytokines had more disease damage, especially renal. High levels of IFN- $\alpha$ were associated with mucocutaneous disease, leukopenia, low complement, Ro/SSA and $\mathrm{La} / \mathrm{SSB}$, whereas vascular events and antiphospholipid antibodies (aPL) were uncommon.

We identified two subgroups with high disease activity: one double IFN- $\lambda 1$ and IFN- $\alpha$ high and another IP-10 high. The former had more neuropsychiatric manifestations, while the latter had more arthritis.

Conclusions Measurements of circulating IFN- $\lambda 1$ and IFN- $\alpha$ define SLE patients with different characteristics. Levels of IFN- $\lambda 1$ correlate with Th17 cytokines and identify a subgroup with more damage. Disease activity is associated with either upregulation of both type I and III IFNs, or independently with IP-10. Our findings could be of major importance when tailoring therapy for SLE patients with agents targeting IFNpathways.

\section{PROINFLAMMATORY CYTOKINES (IL6, TNF $\alpha$ AND INTERFERON $\gamma$ ) IN PATIENTS WITH SYSTEMIC LUPUS ERYTHEMATOSUS AND THEIR CLINICAL CORRELATION \\ ${ }^{1} S$ Sankaran*. ${ }^{1}$ Chennai, India \\ 10.1136/lupus-2017-000215.234}

Background and aims To study the levels of proinflammatory cytokines (IL6, TNFo, IFN- $\gamma$ ) in systemic lupus erythematosus (SLE) patients as compared to healthy control volunteers and to correlate the levels of cytokines with clinical features in Indian patients

Methods After obtaining Institutional ethical clearance and written informed consent, 88 consecutive SLE patients(newly diagnosed and those in remission) and 60 age and sex matched healthy controls were taken for the study, which was conducted between June to December 2015 in a tertiary care centre. Patients who had overlap syndromes, Mixed connective tissue disorder, antiphospholipid antibody syndrome, secondary Sjogrens syndrome, Lupus flare due to infections and offending drugs,were excluded. A thorough history, clinical examination, baseline biochemical and immunological investigations were done. Serum IL6, TNF $\alpha$, IFN- $\gamma$ were estimated in all patients and controls by ELISA. Statistical methods were done using SPSS software.

Results Serum interferon $\gamma$ levels were higher in patients $($ mean $\pm \mathrm{SD}=25.65 \pm 64.81 \mathrm{pg} / \mathrm{ml}$; median 8) than the controls $($ mean $\pm S D=2.95 \pm 10.28 \mathrm{pg} / \mathrm{ml}$, median -0$),(p=0.0080)$. Serum IL6 levels were also higher in patients (mean $\pm \mathrm{SD}=143.01$ $\pm 64.94 \mathrm{pg} / \mathrm{ml}$ ) than controls (mean $\pm \mathrm{SD}=69.33 \pm 11.7 \mathrm{pg} / \mathrm{ml}$ ), $(\mathrm{p}<0.0001)$. Serum TNF- $\alpha$ were also elevated in patients $($ mean $\pm S D=427.13 \pm 206.49 \mathrm{pg} / \mathrm{ml}$; median 384.5$)$ than controls (mean $\pm \mathrm{SD}=236.05 \pm 23.53 \mathrm{pg} / \mathrm{ml}$; median 238), $(\mathrm{p}<0.0001)$. Interferon $\gamma$ levels were significantly higher in females, lower in patients with lymphadenopathy and significantly higher in lupus nephritis class III,positively correlated with thrombocytopenia and negatively correlated with ESR, dsDNA, C3, C4. IL6 and TNF $\alpha$ were significantly associated with oral ulcer and alopecia respectively. Both showed a 
positive correlation with ESR, dsDNA, C3, C4. Cytokines didn't correlate with SLEDAI.

Conclusions IL6 and TNF $\alpha$ are reliable markers of disease activity, but lack significant clinical correlation.

\section{HIERARCHICAL CLUSTER ANALYSIS OF SYSTEMIC LUPUS ERYTHEMATOSUS}

${ }^{1} \mathrm{CH}$ Suh, ${ }^{1} \mathrm{JY}$ Jung, ${ }^{2} \mathrm{HY}$ Lee, ${ }^{1} \mathrm{HA}$ Kim, ${ }^{3} \mathrm{SS} \mathrm{Kim},{ }^{4} \mathrm{~S} J$ Hong. ${ }^{1}$ Ajou University School of Medicine, Rheumatology, Suwon, Republic of Korea; ${ }^{2}$ Ajou University School of Medicine, Clinical Trial Centre, Suwon, Republic of Korea; ${ }^{3}$ Ulsan University College of Medicine, Rheumatology, Gangneung, Republic of Korea; ${ }^{4}$ Kyung Hee University Hospital, Rheumatology, Seoul, Republic of Korea

10.1136/lupus-2017-000215.235

Background and aims Systemic lupus erythematosus (SLE) is a heterogeneous disorder with diverse manifestations and serologic features. The purpose is to categorise SLE patients into similar initial characteristics.

Methods Hierarchical cluster analysis approached to 389 SLE patients and 10 laboratory values. Laboratory values were transformed into Z-score for hierarchical clustering. Ward's method as agglomeration method was a criterion applied with spearman correlation as distance metric. Clinical characteristics among clusters were examined by ANOVA with Tukey and Fisher's exact test. To find each SLE cluster using initial laboratory, linear discriminant analysis was applied.

Results Three clusters were revealed by initial laboratory data; Cluster 1 had higher anti-dsDNA antibody, ANA titer and ESR, and low complements, lymphocyte, haemoglobin and platelet counts, Cluster 2 had lower anti-dsDNA antibody, ANA titer and ESR, and Cluster 3 had lower anti-dsDNA antibody titer, WBC and lymphocyte counts, and higher ANA titer. As a result from analysing cumulative manifestations and treatment, Cluster 1 showed more frequent malar rash, alopecia and renal disease with higher SLEDAI, and more use of cyclophosphamide and azathioprine. Also, oral ulcer was developed frequently in Cluster 2. During disease duration, total and mean corticosteroids and the number of flare were higher in Cluster 1.

Conclusions With initial laboratory values, SLE patients could be divided 3 clusters. Each Cluster showed different characteristics in clinical manifestations and treatment patterns. This predictive model considered disease severity had $84.6 \%$ of total predictability.

\section{ANTI-NUCLEAR ANTIBODY PATTERN AND CLINICAL MANIFESTATION PRESENTATION IN SYSTEMIC LUPUS ERYTHEMATOSUS}

${ }^{1}$ BS Suryajaya, 'VN Moenardi, ${ }^{1}$ II Hidayat, ${ }^{2}$ L Hamijoyo. ${ }^{1}$ Faculty Of Medicine Padjadjaran University/Hasan Sadikin General Hospital, Immunology study group, Bandung, Indonesia; ${ }^{2}$ Faculty Of Medicine Padjadjaran University/Hasan Sadikin General Hospital, Immunology study group Rheumatology Division- Internal Medicine Department, Bandung, Indonesia

\subsection{6/lupus-2017-000215.236}

Background and aims Anti-nuclear antibody (ANA) pattern analysis by immunofluorescence microscopy remains an important diagnostic tools for Systemic Lupus Erythematosus (SLE) in Indonesia. Although the utilisation of ANA pattern are used by physician to diagnosed SLE, there are very sparse study to explain on SLE clinical presentation and ANA pattern in Indonesia. This study was compiled to determined ANA pattern and clinical manifestation presentation in ANA positive patients.

Methods This study was an observational cross-sectional study in Hasan Sadikin General Hospital Bandung, 217 patients that had satisfied American College of Rheumatology (ACR) Criteria (1997) and ANA pattern were positive when diagnosed with SLE. Data was acquired from "RSHS Lupus Registry" database.

Results The study population consist of 217 patients, whom 208 patients (96\%) were female and 206 patients (96\%) were Sundanese (median age was 33, ranging from age 14 to 62 years), had Speckled ANA pattern (S-ANA) 98 patients (45\%), Homogenous ANA Pattern (H-ANA) 63 patients (29\%), Nucleolar ANA Pattern (N-ANA) 23 patients (11\%), SpeckledNucleolar ANA Pattern (SN-ANA) 18 patients (8\%), and other staining patterns 15 patients (7\%).The majority of clinical manifestation in S-ANA and H-ANA patients were haematological involvement with 69 patients (70\%) and 42 patients (67\%) respectively, and N-ANA and SN-ANA were malar rash with 20 patients (87\%), and 13 patients (72\%) respectively.

Conclusions The most frequent ANA pattern among SLE patients in this study is S-ANA pattern. The most common clinical presentation found is haematological involvement in SANA and H-ANA and malar rash in N-ANA and SN-ANA.

\section{ISCHAEMIC STROKE IN SYSTEMIC LUPUS ERYTHEMATOSUS, -DISTRIBUTION OF SUBTYPES AND A RISK GENOTYPE IN THE STAT4 GENE}

${ }^{1} \mathrm{E}$ Svenungsson* ${ }^{2} \mathrm{~L}$ Hopia, ${ }^{3} \mathrm{~A}$ Laveskog, ${ }^{4} \mathrm{~A}$ Jönsen, ${ }^{5} \mathrm{D}$ Leonard ${ }^{1} \mathrm{JT}$ Gustafsson, ${ }^{1}$ I Gunnarsson, ${ }^{6} \mathrm{~A}$ Zickert, ${ }^{5} \mathrm{G}$ Nordmark, ${ }^{4} \mathrm{AA}$ Bengtsson, ${ }^{7} \mathrm{~K}$ Elvin, ${ }^{8} \mathrm{JK}$ Sandling, ${ }^{8} \mathrm{AC}$ Syvänen, ${ }^{5} \mathrm{~L}$ Rönnblom, ${ }^{2} \mathrm{M}$ Andersson. ${ }^{1}$ Karolinska Institutet, Unit of Rheumatology- Department of Medicine- Solna, Stockholm, Sweden; ${ }^{2}$ Karolinska Institutet, Department of Clinical Neuroscience, Stockholm, Sweden; ${ }^{3}$ Karolinska Institutet, Department of Neuroradiology, Stockholm, Sweden; ${ }^{4}$ Lund University, Department of Clinical SciencesSection of Rheumatology, Lund, Sweden; ${ }^{5}$ Uppsala University, Department of Medical Sciences- Section of Rheumatology, Uppsala, Sweden; ${ }^{6}$ Karolinska Institutet, Unit of Rheumatology- Department of Medicine- Huddinge, Stockholm, Sweden; ${ }^{7}$ Karolinska Institutet, Unit of Clinical Immunology- Department of Clinical Immunology and Transfusion Medicine-, Stockholm, Sweden; ${ }^{8}$ Uppsala University, Department of Medical SciencesMolecular Medicine, Uppsala, Sweden

\subsection{6/lupus-2017-000215.237}

Background and aims We investigated the distribution of ischaemic stroke subtypes, classified according the Trial of Org 10172 in Acute Stroke Treatment (TOAST) system, among patients with systemic lupus erythematosus (SLE). Genetic susceptibility in the signal transducer and activator of transcription factor 4 (STAT4) gene, defined by the single nucleotide polymorphism (SNP) rs10181656(G) were explored.

Methods We identified 69/665 SLE patients with stroke. Medical charts were retrieved and brain, cardiac and vascular imaging at the time of stroke were examined. Classification was performed according to TOAST: large-artery atherosclerosis (LAA), cardioembolism (CE), small-artery occlusion (SAO), stroke of other determined aetiology (OC) and stroke of undetermined aetiology (UE). Occurrence of the anti-phospholipid syndrome (APS) was documented. Evaluators were blinded to genotypes. General population controls $(n=658)$ and SLE patients free from previous cerebrovascular disease $(n=517)$ were used as comparators. 